\title{
Esophageal cooling for protection during left atrial ablation: a systematic review and meta-analysis
}

\author{
Lisa WM Leung ${ }^{1} \cdot$ Mark M Gallagher $^{1} \cdot$ Pasquale Santangeli $^{2} \cdot$ Cory Tschabrunn $^{2}$ - Jose M Guerra ${ }^{3}$ - Bieito Campos ${ }^{3}$. \\ Jamal Hayat ${ }^{4} \cdot$ Folefac Atem $^{5} \cdot$ Steven Mickelsen $^{6} \cdot$ Erik Kulstad $^{7}$ (I)
}

Received: 13 September 2019 / Accepted: 31 October 2019 / Published online: 22 November 2019

(C) The Author(s) 2019

\begin{abstract}
Purpose Thermal damage to the esophagus is a risk from radiofrequency (RF) ablation of the left atrium for the treatment of atrial fibrillation (AF). The most extreme type of thermal injury results in atrio-esophageal fistula (AEF) and a correspondingly high mortality rate. Various strategies for reducing esophageal injury have been developed, including power reduction, esophageal deviation, and esophageal cooling. One method of esophageal cooling involves the direct instillation of cold water or saline into the esophagus during RF ablation. Although this method provides limited heat-extraction capacity, studies of it have suggested potential benefit. We sought to perform a meta-analysis of published studies evaluating the use of esophageal cooling via direct liquid instillation for the reduction of thermal injury during RF ablation.

Methods We searched PubMed for studies that used esophageal cooling to protect the esophagus from thermal injury during RF ablation. We then performed a meta-analysis using a random effects model to calculate estimated effect size with $95 \%$ confidence intervals, with an outcome of esophageal lesions stratified by severity, as determined by post-procedure endoscopy.

Results A total of 9 studies were identified and reviewed. After excluding preclinical and mathematical model studies, 3 were included in the meta-analysis, totaling 494 patients. Esophageal cooling showed a tendency to shift lesion severity downward, such that total lesions did not show a statistically significant change (OR 0.6, 95\% CI 0.15 to 2.38). For high-grade lesions, a significant OR of 0.39 (95\% CI 0.17 to 0.89 ) in favor of esophageal cooling was found, suggesting that esophageal cooling, even with a low-capacity thermal extraction technique, reduces the severity of lesions resulting from RF ablation.
\end{abstract}

Erik Kulstad

Erik.Kulstad@UTSouthwestern.edu

Lisa WM Leung

Lisa.Leung@stgeorges.nhs.uk

Mark M Gallagher

Mark.Gallagher@stgeorges.nhs.uk

Pasquale Santangeli

pasquale.santangeli@uphs.upenn.edu

Cory Tschabrunn

cory.tschabrunn@uphs.upenn.edu

Jose M Guerra

jguerra@secardiologia.es

Bieito Campos

bcamposg@santpau.cat

Jamal Hayat

jamal.Hayat@stgeorges.nhs.uk

Folefac Atem

Folefac.Atem@UTSouthwestern.edu
Steven Mickelsen

mickelsenone@gmail.com

1 Department of Cardiology, St George's University Hospitals NHS Foundation Trust, St. George's, University of London, Cranmer Terrace, Tooting, London SW17 0RE, UK

2 Perelman School of Medicine, University of Pennsylvania, 3400 Civic Center Blvd, PA 19104 Philadelphia, United States

3 Hospital de la Santa Creu I Sant Pau, Universitat Autònoma de Barcelona, CIBERCV, Carrer de Sant Quintí, 89, 08041 Barcelona, Spain

4 Department of Gastroenterology, St George's University Hospitals NHS Foundation Trust, St. George's, University of London, Cranmer Terrace, Tooting, London SW17 0RE, UK

5 Department of Neurology and Neurotherapeutics, UT Southwestern Medical Center, 5323 Harry Hines Blvd, 75390 Dallas, TX, United States

6 Department of Internal Medicine, University of Iowa Carver College of Medicine, 200 Hawkins Drive, 52242 Iowa City, United States

7 Department of Emergency Medicine, UT Southwestern Medical Center, 5323 Harry Hines Blvd, 75390 Dallas, TX, United States 
Conclusions Esophageal cooling reduces the severity of the lesions that may result from RF ablation, even when relatively low heat extraction methods are used, such as the direct instillation of small volumes of cold liquid. Further investigation of this approach is warranted, particularly with higher heat extraction capacity techniques.

Keywords Atrial fibrillation $\cdot$ Radiofrequency ablation $\cdot$ Esophageal injury $\cdot$ Esophageal cooling $\cdot$ Atrio-esophageal fistula

\section{Introduction}

Thermal damage to the esophagus is a risk from radiofrequency (RF) ablation or cryoablation of the left atrium for the treatment of atrial fibrillation (AF) [1-3]. The most extreme type of thermal injury is an atrioesophageal fistula (AEF), with a mortality rate of $80 \%$ or more [4-8]. Various strategies for protecting the esophagus during RF ablation or reducing the severity of injury have been developed, including power reduction, avoidance of greater contact force, temperature monitoring, esophageal deviation, and esophageal cooling, with varying degrees of success [9-11].

Esophageal cooling for the purpose of protecting the esophagus during RF ablation has been investigated in multiple studies [12-20]. The techniques used have included the insertion of expandable balloon devices or cooling sacs that circulate water, and the direct instillation of ice-cold water or saline into the esophagus. The study designs have included animal models and mathematical models as well as human clinical studies. Most of the human clinical studies have used direct instillation of ice cold water or saline as the cooling method, and for this reason, we performed a meta-analysis of the data obtained in these studies to examine their range of effect sizes and estimate the potential efficacy of esophageal cooling for protection during RF ablation.

\section{Methods}

\subsection{Data sources and search strategy}

Using PubMed, we searched the literature dated from 1985 (prior to the earliest reports of endocardial ablation to treat atrial fibrillation) to June 2019 for studies published on esophageal cooling during cardiac ablation. We conducted a broad search with the following Boolean structure: (esophag* OR oesophag*) AND cooling AND (ablation OR fibrillation). We did not restrict the search to studies published in English only. Details of the systematic review were submitted for registration in PROSPERO on June 21, 2019, with further details describing the statistical plan added on September 11, 2019.

\subsection{Eligibility criteria}

We excluded preclinical studies, bench-top, agar phantom, and mathematical model studies, and studies that did not include formal endoscopy as an outcome measure.

\subsection{Data collection}

The primary data of interest were esophageal lesions identified endoscopically after RF ablation. Because we anticipated inconsistency in the categorization of lesion severity, we aimed to simplify all lesion severity measurement into severe lesions characterized by the presence of ulceration and mild to moderate lesions encompassing all other abnormalities. Studies identified were then assessed for quality using the Newcastle Ottawa Scale, which evaluates three quality parameters (selection, comparability, and outcome) divided across eight specific items. Each item on the scale is scored with up to one point, except for comparability, which can be adapted to the specific topic of interest to score up to two points, such that the maximum for each study is 9 , with studies having less than 5 points being identified as representing a high risk of bias [21].

\subsection{Statistical analysis}

For this meta-analysis, we input the study data into Review Manager 5.3, and we present the results graphically. SAS version 9.4 (SAS Institute Inc., Cary, NC, USA) was used for additional analyses. The Cochran-Mantel-Haenszel (CMH) method was employed to test the null hypothesis that the response rate is the same for the two arms (control versus treatment), after adjusting for possible differences in study response rates. Furthermore, we fitted a random effect model using SAS procedures GLIMMIX and NLMIXED by treating the studies as a random effect. Because lesion grades are often considered to be dichotomized into those that are likely to progress to AEF and those that are not, we initially analyzed the data as a binary outcome (high-grade lesions concerning for progression versus low grade lesions likely to heal spontaneously). Then, to further estimate effect size, we used an ordinal logistic random intercept model, taking into account the ordered nature of lesion grading (low to high, numerically). 


\section{Results}

We identified 9 studies using the above criteria. Five of these studies were excluded for being non-clinical. Berjano et al. utilized a finite element model in three dimensions to investigate the effects of a cooled intraesophageal balloon [12]. Lequerica et al. performed studies using an agar phantombased model that was built to provide temperature readings at points between the esophageal lumen and the myocardium $[13,14]$. Arruda et al. studied a custom developed system utilizing temperature-controlled saline or water in an in vitro lamb heart and esophagus preparation, followed by an in vivo model with six dogs [15]. Scanavacca et al. presented a study of the use of a saline filled esophageal balloon to attempt esophageal protection in a dog model [17]. A clinical study of 8 patients by Tsuchiya et al. was excluded for not using endoscopy to determine the presence of lesions after RF ablation [16].

The remaining 3 studies included a total of 494 patients. Details of these studies and the characteristics of the patients are shown in Tables 1, 2, 3.Because the manner of grading lesions varied among the studies, we incorporated the scales used in the three studies into a common stratification (Grades I, II, III, and IV).

John et al. studied 76 patients, half of which were actively cooled by injecting a $20 \mathrm{~mL}$ bolus of ice-cold saline via orogastric tube into the upper esophagus if/when the luminal esophageal temperature (LET) increased by $0.5^{\circ} \mathrm{C}$ above baseline [20]. The authors found that this method of esophageal cooling did not decrease the overall incidence of thermal lesions, but noted a trend toward fewer severe lesions with cooling (Fig 1). The authors graded lesions as follows: grade 0 , no esophageal lesion; grade 1 , mucosal damage $<1 \mathrm{~cm}$ width; grade 2, mucosal damage 1-3 cm width; grade 3 , mucosal damage $>3 \mathrm{~cm}$ width or visualization of deeper layer; and grade 4, bleeding ulcer or with overlying clot. Assessment of the study quality resulted in a score of 8 using the Newcastle Ottawa Scale.

Kuwahara et al. studied 100 patients using very small volumes $(5 \mathrm{~mL})$ of ice water as the coolant for half of them. This volume was injected prior to RF energy delivery as well as subsequently if/when the LET reached $42{ }^{\circ} \mathrm{C}$ [18]. The authors found that this approach reduced the severity of esophageal lesions, but did not reduce the incidence: lesions occurred in $20 \%$ of the treatment group and $22 \%$ of the controls, with 3 moderate and 7 mild in the cooled group and 3 severe, 1 moderate, and 7 mild in the control group (Fig 2). The authors graded the severity of the lesions as mild, moderate, or severe, according to their extent and color. Assessment of the study quality resulted in a score of 8 using the Newcastle Ottawa Scale.

Sohara et al. studied 318 consecutive patients divided into three groups, one receiving only temperature monitoring 


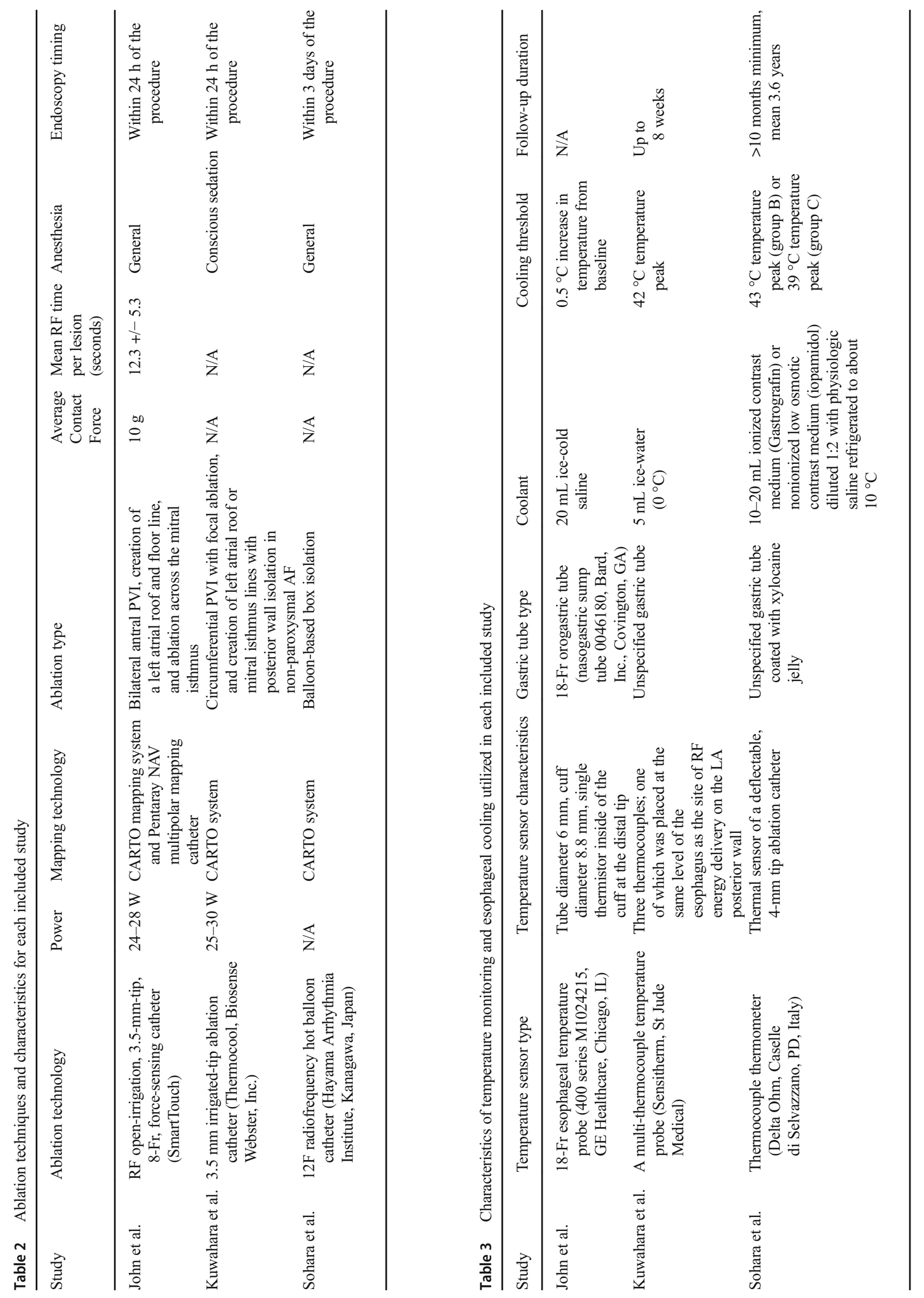


Fig. 1 Results from John et al. Patients in the treatment group were actively cooled by injecting a $20 \mathrm{~mL}$ bolus of ice-cold saline via orogastric tube into the upper esophagus if/when the LET increased by $0.5^{\circ} \mathrm{C}$ above baseline. Grade III and grade IV lesions are shown separately

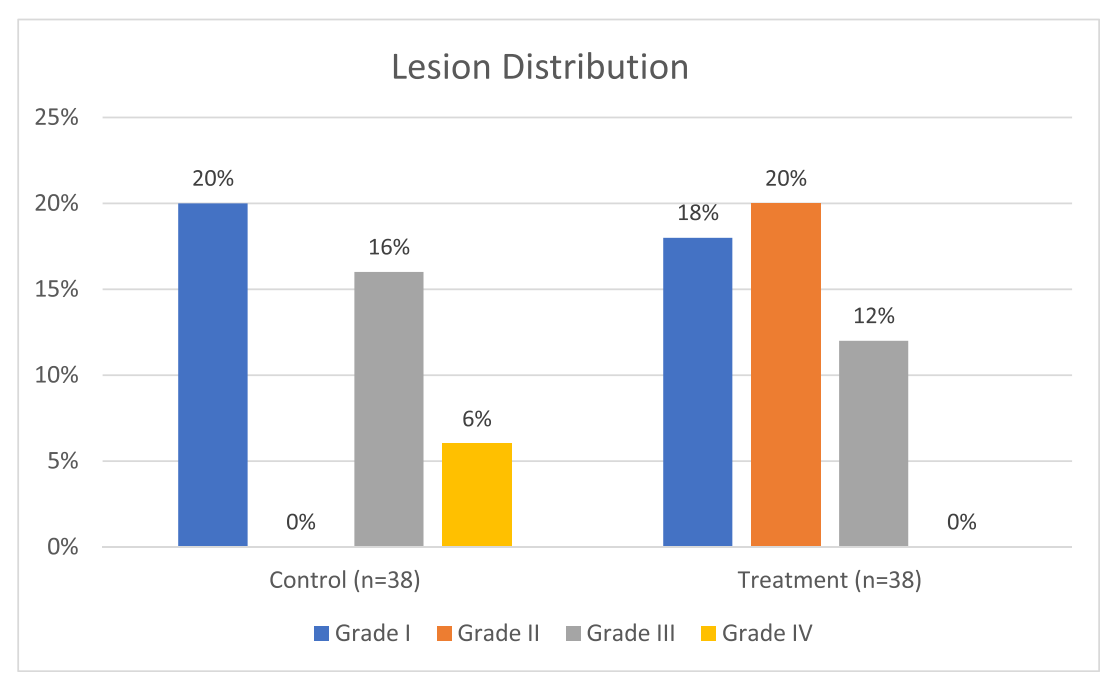

without cooling, the second receiving temperature monitoring with cooling when the LET exceeded $43{ }^{\circ} \mathrm{C}$, and the third receiving temperature monitoring with cooling when the LET exceeded $39^{\circ} \mathrm{C}$. These authors used cooled saline mixed with Gastrografin or iopamidol as the coolant. The total volumes injected were slightly higher than those used by John et al. and Kuwahara et al. but were still limited $(10-20 \mathrm{~mL}$ in repeated injected aliquots with a temperature of approximately 10 $\left.{ }^{\circ} \mathrm{C}\right)$.[19] The percentage of patients free from any ulceration or erosion in each group was found to be $63.6 \%, 87.5 \%$, and $95.2 \%$, respectively (Fig 3). The authors classified the lesions as normal (score 1), erosion (patchy mucosal ulceration: score 2), mild ulcer (necrosis less than $3 \mathrm{~mm}$ in diameter with red spot: score 3), severe ulcer (necrosis more than $3 \mathrm{~mm}$ in diameter with red spot and/or with a hemorrhagic appearance, often with fibrinoid material: score 4). Assessment of the study quality resulted in a score of 9 using the Newcastle Ottawa Scale.
The studies by Kuwahara et al. and John et al. show a clear shift from high-grade to lower-grade lesions between the control and treatment arms $[18,20]$. In contrast, the data from Sohara et al. show a general reduction in lesions of all grades [19].

The forest plot comparing the outcome of all lesions (grades I, II, III, and IV) as events between control and treatment arms is shown in Fig 4. Although fewer lesions occurred in the treatment arms of the Sohara et al. and Kuwahara et al. studies, in meta-analysis this decrease did not reach statistical significance (OR 0.6, 95\% CI 0.15 to 2.38 ).

The slight increase in low-grade lesions seen in the John et al. and Kuwahara et al. studies is shown in the forest plot in Fig 5, with an OR of 1.0 (95\% CI 0.26 to 3.93). The number of low-grade lesions is not significantly impacted with this treatment.
Fig. 2 Results from Kuwahara et al. Patients in the treatment group were actively cooled by injecting $5 \mathrm{~mL}$ of ice water prior to RF energy delivery and subsequently when the LET reached $42{ }^{\circ} \mathrm{C}$. The Grade III/IV lesion category represents all lesions qualitatively graded as "severe," with mild lesions in Grade I and moderate lesions in Grade II

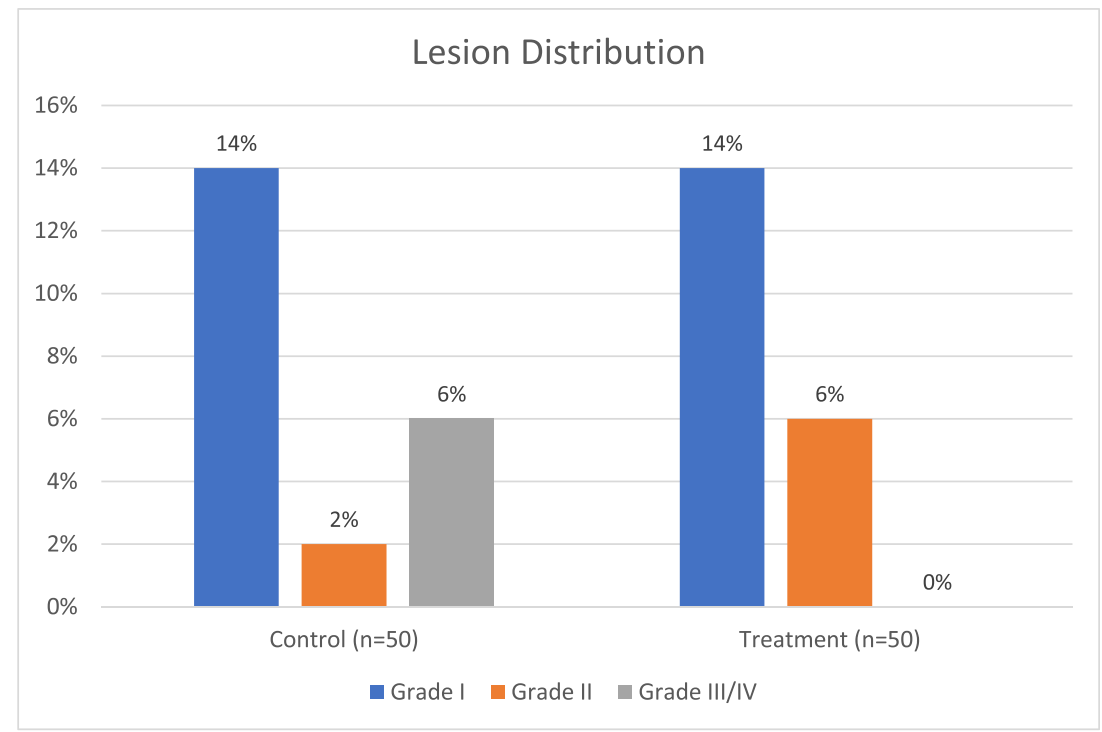


Fig. 3 Results from Sohara et al. Patients in group A received only LET monitoring without cooling of the esophagus. Patients in groups B and C received LET monitoring with esophageal cooling when the LET exceeded $43{ }^{\circ} \mathrm{C}$ and $39^{\circ} \mathrm{C}$, respectively. Cooling was by infusion of cooled saline mixed with Gastrografin. The Grade III/IV lesion category represents all lesions graded as ulcers (scored as 3 or 4 by Sohara et al.)

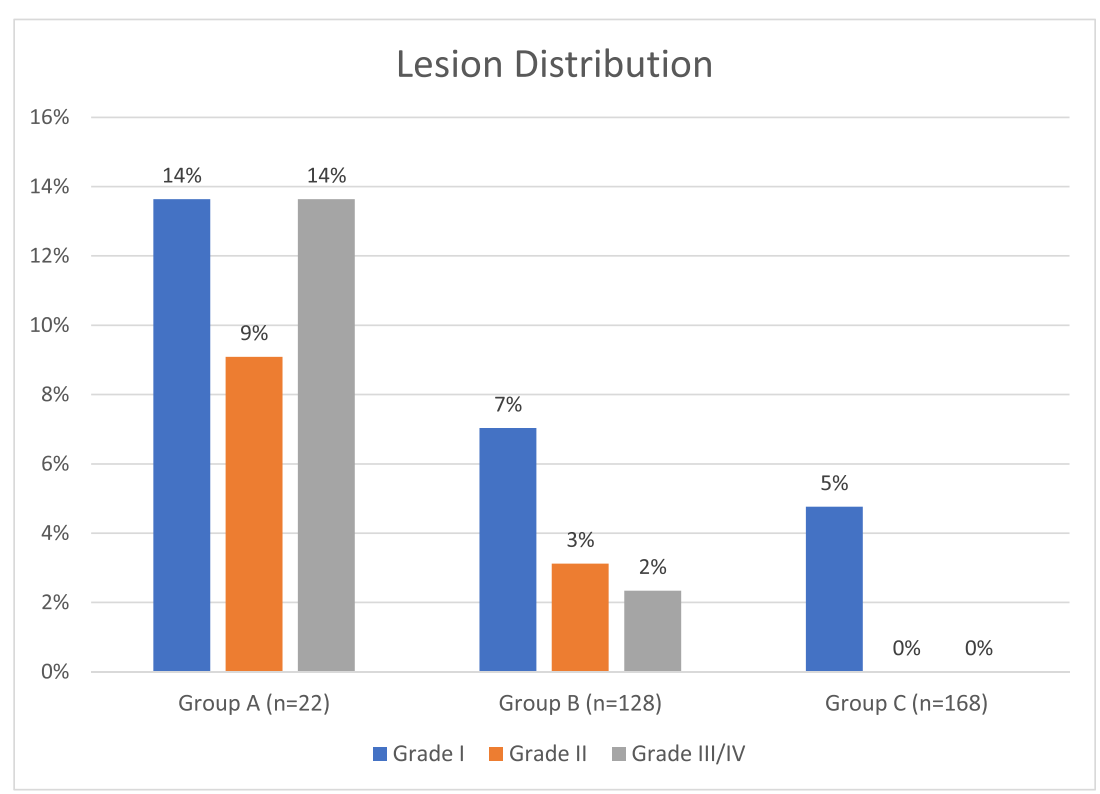

Evaluating the occurrence of high-grade lesions (grade III and IV) results in the forest plot shown in Fig 6, demonstrating a significant OR of 0.39 (95\% CI 0.17 to 0.89 ) in favor of the treatment arm. Separately, using the CMH method, we obtained a significant $p$ value of 0.016 indicating that the association between treatment and lesion grade remains strong. Furthermore, in a binary logistic regression model, an OR of $0.46(95 \%$ CI 0.28 to 0.75$)$ was found.

The $\mathrm{I}^{2}$ statistic, which describes the percentage of total variation across studies that is due to heterogeneity rather than chance, is shown in each of Figs. 4, 5, and 6. For the analysis using an outcome of severe lesions (Fig. 6), the $\mathrm{I}^{2}=0 \%$, which indicates no observed heterogeneity. Increasing heterogeneity of effect is seen (shown in the forest plots) when looking at the outcome of all lesions, and low-grade lesions, in Figs. 4 and 5, respectively.

Finally, using an ordinal logistic random intercept model rather than dichotomized outcome, we used GLIMMIX and NLMIXED in SAS to obtain an additional estimate of effect size for each category of lesion independently. This method showed that esophageal cooling by the method of direct instillation of cold water or saline used in these studies results in a point estimate of a $-23 \%$ reduction in lesion grade, with $95 \%$ CI ranging from $-85 \%$ to $+38 \%$.

\section{Discussion}

Esophageal injury from RF ablation remains a feared complication in the treatment of atrial fibrillation, and a variety of techniques have been developed to reduce this risk. Esophageal cooling has shown promise in a number of studies, with such cooling being brought about by the instillation of cold water or saline directly into the esophagus via orogastric tube. Even with the relatively high heat capacity of water, the amount of thermal energy that can actually be absorbed by this method is limited by the low total volumes of liquid instilled. Nevertheless, our meta-analysis of three studies that used this approach suggests that cooling in this manner offers a clinically significant protective effect from severe lesions, and provides a $61 \%$ reduction in high-grade lesion formation (with a $95 \%$ CI of $11 \%$ to $83 \%$ reduction).

Prototypes of various balloon devices have been evaluated for possible use in preventing thermal injury during RF

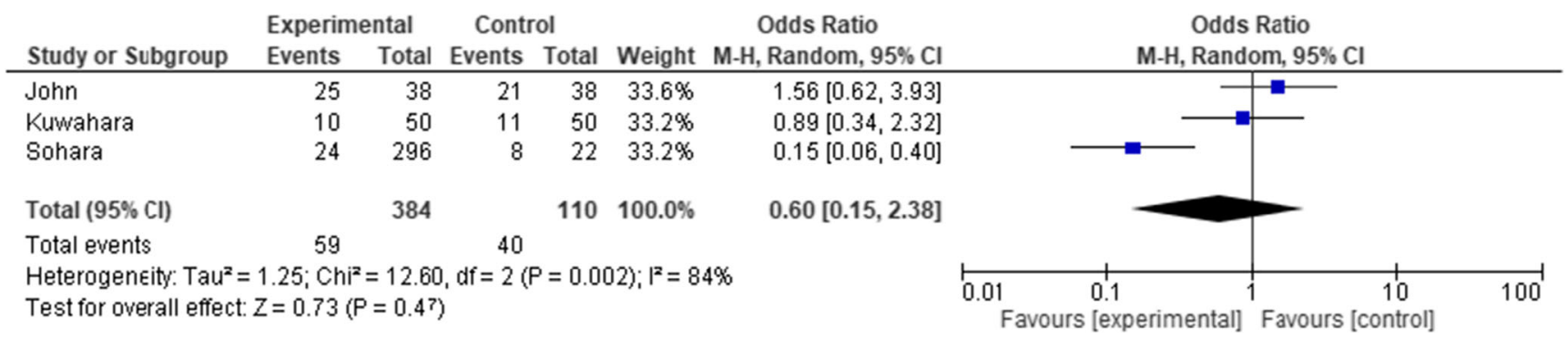

Fig. 4. Forest plot comparing the outcome of all lesions in the three clinical studies. Events are the occurrence of grade I, II, III, and IV lesions 


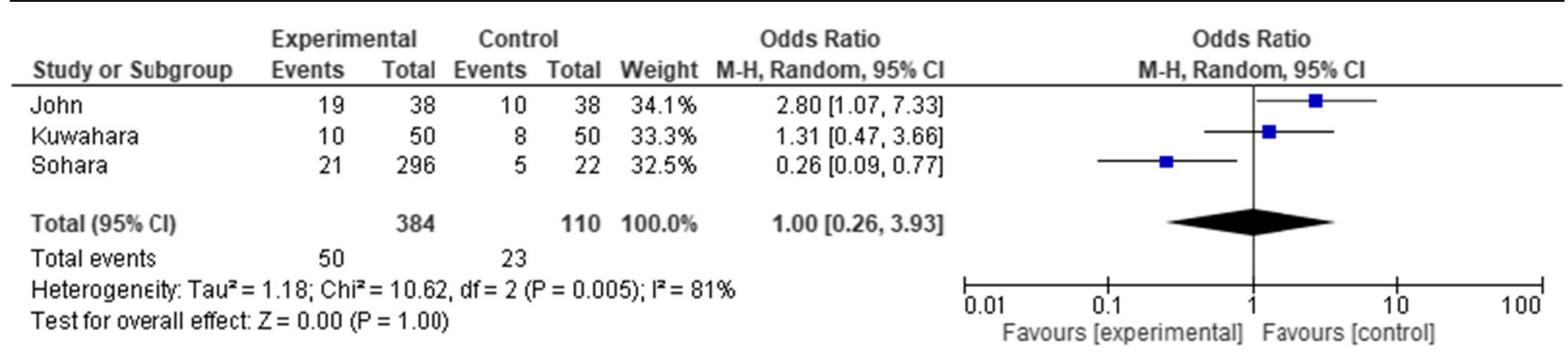

Fig. 5. Forest plot comparing the outcome of low-grade lesions in the three clinical studies. Events are the occurrence of grade I and II lesions

ablation [13-16]. These devices provided flow rates of $25 \mathrm{~mL}$ to $300 \mathrm{~mL}$ per minute, and although preclinical as well as mathematical models of the devices suggested benefits in lesion reduction, none of the early prototypes evolved into commercially available products. A more recently developed intraesophageal cooling device has a flow rate of up to 1900 $\mathrm{mL}$ per minute. This device was designed for whole-body temperature manipulation, for which it is commercially available, and has been shown to have protective effects in animal and mathematical models of RF ablation. It is currently under clinical investigation for use during RF ablation, and preclinical data suggest a close correlation between water temperature and protective effect, such that increased heat extraction results in greater reduction in lesion thickness $[22,23]$. Recent data in the burn literature also suggests that there are improved outcomes from thermal burns (reduced full thickness depth, skin grafting requirement, hospitalization, and other operative interventions) with cooling, with a dose-response relationship noted between the odds of grafting and duration of cool running water, which may offer further support for the idea of a threshold effect to preventing progression of thermal injury after the initial thermal insult [24].

A recent meta-analysis of two studies that used the direct instillation of ice-cold water to cool the esophagus focused on the outcome of overall lesion frequency, but did not distinguish between lesion severity and lesion frequency, and produced inconclusive results [25]. In contrast, in our meta-analysis of 3 studies that used this cooling method, we stratified the lesions according to their severity and found that cooling by this method reduced the number of high-grade lesions. Although the mechanism of AEF formation is not well understood, there is general agreement that thermal injury is a precursor and that higher-grade thermal injury has a higher risk of progression to AEF [26].

Analyzing each lesion grade independently in a statistical model allows an alternative approach to estimate effect size that may provide further refinement of the estimate, at the cost of decreased precision of the estimate. The point estimate that we found using this approach suggests a reduction in highgrade lesions of $-23 \%$, although a higher number of patients with a greater number of high-grade lesions would be necessary to narrow the confidence intervals around this estimate, which, with the population included here, ranges from $-85 \%$ to $+38 \%$. It seems likely that cooling methods that have a higher heat extraction capacity will lead to increased effect size point estimates when the data are analyzed by either binary logistic or ordinal regression.

\section{Limitations}

The three studies that we analyzed differed in patient characteristics and other details as well as in the specific radiofrequency techniques and equipment used (see Tables 1, 2, 3). Nevertheless, all studies used radiofrequency ablation, and the variation in technology reflects current real-world practice. One of the studies randomized the patients. There was no description of any attempt at blinding the patients to the protection strategy used. Lesion grading varied between studies, but the scales used in each study fitted easily into a common stratification. The differences between the studies may serve to broaden the generalizability of this meta-analysis.

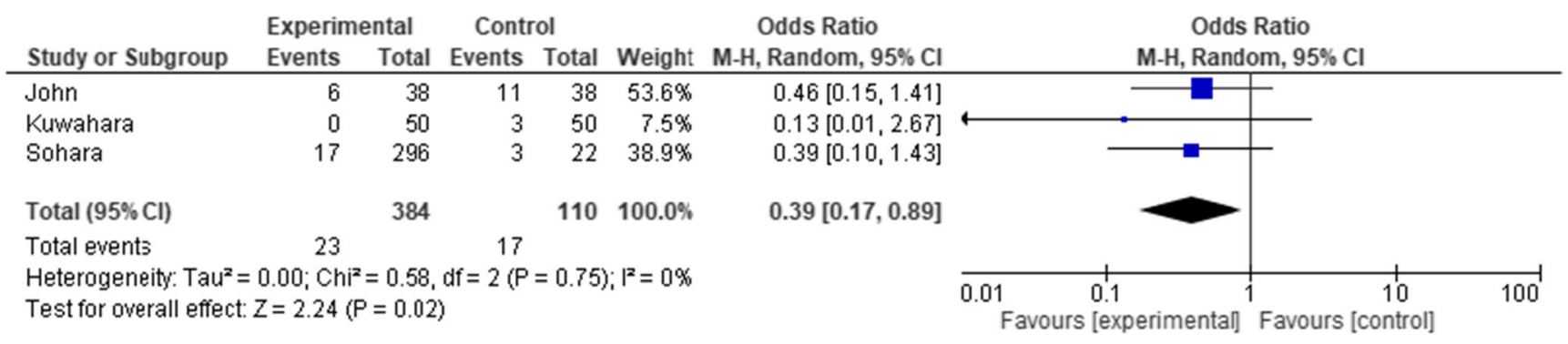

Fig. 6 Forest plot comparing the outcome of severe lesions. Events are the occurrence of grade III/IV lesions 


\section{Conclusions}

Esophageal cooling reduces the severity of the lesions that may result from RF ablation, even when relatively low heat extraction methods are used, such as the direct instillation of small amounts of cold liquid. Further investigation of esophageal cooling is warranted, particularly with higher heat extraction capacity techniques.

Author contributions LL: design, data acquisition, data analysis/interpretation, manuscript drafting; MG: concept, data analysis/interpretation, manuscript drafting; PS: data analysis/interpretation, manuscript drafting; CT: data analysis/interpretation, manuscript drafting; JG: data analysis/interpretation, manuscript drafting; $\mathrm{BC}$ : data analysis/interpretation, manuscript drafting and revisions; JH: data analysis/interpretation, endoscopy expertise, manuscript review; FA: statistical analysis, data analysis/interpretation; SM: data analysis/ interpretation, manuscript revisions; EK: concept, design, data analysis/interpretation, manuscript drafting. All authors provided critical review, revision, and approval of the manuscript.

\section{Compliance with ethical standards}

Conflict of interest EK declares equity interest in Attune Medical, manufacturer of an esophageal cooling device; MG, PS, CT, JG, and BC serve as Principal Investigators for studies of esophageal cooling sponsored by Attune Medical, which includes institutional support and travel reimbursements. SM has provided consulting services for Attune Medical. All other authors declare no relevant conflicts of interest.

Open Access This article is distributed under the terms of the Creative Commons Attribution 4.0 International License (http:// creativecommons.org/licenses/by/4.0/), which permits unrestricted use, distribution, and reproduction in any medium, provided you give appropriate credit to the original author(s) and the source, provide a link to the Creative Commons license, and indicate if changes were made.

\section{References}

1. Romero J, Avendano R, Grushko M, Diaz JC, Du X, Gianni C, et al. Oesophageal injury during AF ablation: techniques for prevention. Arrhythm Electrophysiol Rev. 2018;7(1):24-31.

2. Kapur S, Barbhaiya C, Deneke T, Michaud GF. Esophageal injury and atrioesophageal fistula caused by ablation for atrial fibrillation. Circulation. 2017;136(13):1247-55.

3. Qumseya BJ, Kusumoto F, Wolfsen H. Esophageal injury following left atrial ablation. Gastroenterol Hepatol (N Y). 2012;8(6): 414-6.

4. Khakpour H, Shemin RJ, Lee JM, Buch E, Boyle NG, Shivkumar $\mathrm{K}$, et al. Atrioesophageal fistula after atrial fibrillation ablation: a single center series. Journal of atrial fibrillation. 2017;10(3):1654.

5. Yousuf T, Keshmiri H, Bulwa Z, Kramer J, Sharjeel Arshad HM, Issa $\mathrm{R}$, et al. Management of atrio-esophageal fistula following left atrial ablation. Cardiol Res. 2016;7(1):36-45.

6. Khan MY, Siddiqui WJ, Iyer PS, Dirweesh A, Karabulut N. Left atrial to esophageal fistula: a case report and literature review. The American journal of case reports. 2016;17:814-8.

7. Nair GM, Nery PB, Redpath CJ, Lam BK, Birnie DH. Atrioesophageal fistula in the era of atrial fibrillation ablation: a review. Can J Cardiol. 2014;30(4):388-95.
8. Zakaria A, Hipp K, Battista N, Tommolino E, Machado C: Fatal esophageal-pericardial fistula as a complication of radiofrequency catheter ablation. SAGE open medical case reports 2019, 7: $2050313 \times 19841150$.

9. Deviating the esophagus in atrial fibrillation ablation - clinicaltrials. gov listing [https://clinicalt rials.gov/ct2/show/NCT01546168]. Accessed 13 Sep 2019

10. Kadado AJ, Akar JG, Hummel JP. Luminal esophageal temperature monitoring to reduce esophageal thermal injury during catheter ablation for atrial fibrillation: a review. Trends in Cardiovascular Medicine. 2019;29(5):264-71.

11. Deneke T, Bunz K, Bastian A, Pasler M, Anders H, Lehmann R, et al. Utility of esophageal temperature monitoring during pulmonary vein isolation for atrial fibrillation using duty-cycled phased radiofrequency ablation. J Cardiovasc Electrophysiol. 2011;22(3): 255-61.

12. Berjano EJ, Hornero F. A cooled intraesophageal balloon to prevent thermal injury during endocardial surgical radiofrequency ablation of the left atrium: a finite element study. Phys Med Biol. 2005;50(20):N269-79.

13. Lequerica JL, Berjano EJ, Herrero M, Hornero F. Reliability assessment of a cooled intraesophageal balloon to prevent thermal injury during RF cardiac ablation: an agar phantom study. J Cardiovasc Electrophysiol. 2008;19(11):1188-93.

14. Lequerica JL, Berjano EJ, Herrero M, Melecio L, Hornero F. A cooled water-irrigated intraesophageal balloon to prevent thermal injury during cardiac ablation: experimental study based on an agar phantom. Phys Med Biol. 2008;53(4):N25-34.

15. Arruda MS, Armaganijan L, Di Biase L, Rashidi R, Natale A. Feasibility and safety of using an esophageal protective system to eliminate esophageal thermal injury: implications on atrialesophageal fistula following AF ablation. J Cardiovasc Electrophysiol. 2009;20(11):1272-8.

16. Tsuchiya T, Ashikaga K, Nakagawa S, Hayashida K, Kugimiya H. Atrial fibrillation ablation with esophageal cooling with a cooled water-irrigated intraesophageal balloon: a pilot study. J Cardiovasc Electrophysiol. 2007;18(2):145-50.

17. Scanavacca MI, Pisani CF, Neto S, Tamaki W, Santo SR, Guirao C, et al. Cooled intra-esophageal balloon to prevent thermal injury of esophageal wall during radiofrequency ablation. In: In: ESC Congress 2007, 1 - 5 September. vol. 28. Vienna, Austria, vol. $156 ; 2007$

18. Kuwahara T, Takahashi A, Okubo K, Takagi K, Yamao K, Nakashima E, et al. Oesophageal cooling with ice water does not reduce the incidence of oesophageal lesions complicating catheter ablation of atrial fibrillation: randomized controlled study. Europace. 2014;16(6):834-9.

19. Sohara H, Satake S, Takeda H, Yamaguchi Y, Nagasu N. Prevalence of esophageal ulceration after atrial fibrillation ablation with the hot balloon ablation catheter: what is the value of esophageal cooling? J Cardiovasc Electrophysiol. 2014;25(7):686-92.

20. John J, Garg L, Orosey M, Desai T, Haines DE, Wong WS. The effect of esophageal cooling on esophageal injury during radiofrequency catheter ablation of atrial fibrillation. J Interv Card Electrophysiol. 2019.

21. Luchini C, Stubbs B, Solmi M, Veronese N. Assessing the quality of studies in meta-analyses: Advantages and limitations of the Newcastle Ottawa Scale. World J Meta-Anal. 2017;5(4):80-4.

22. Montoya MM, Mickelsen S, Clark B, Arnold M, Hanks J, Sauter E, et al. Protecting the esophagus from thermal injury during radiofrequency ablation with an esophageal cooling device. Journal of atrial fibrillation. 2019;11(5):2110.

23. Feher M, Anneken L, Gruber M, Achenbach S, Arnold M. Esophageal cooling for prevention of thermal lesions during left atrial ablation procedures: a first in man case series. In: In: EHRA 2019: March 19, 2019 2018; Lisbon, Portugal; 2018. 
24. Griffin BR, Frear CC, Babl F, Oakley E, Kimble RM. Cool running water first aid decreases skin grafting requirements in pediatric burns: a cohort study of two thousand four hundred ninety-five children. Ann Emerg Med. 2019.

25. Ha FJ, Han HC, Sanders P, Teh AW, O'Donnell D, Farouque O, et al. Prevalence and prevention of oesophageal injury during atrial fibrillation ablation: a systematic review and meta-analysis. Europace. 2019;21(1):80-90.
26. Halbfass P, Pavlov B, Muller P, Nentwich K, Sonne K, Barth S, et al. Progression from esophageal thermal asymptomatic lesion to perforation complicating atrial fibrillation ablation: a single-center registry. Circ Arrhythm Electrophysiol. 2017;10(8).

Publisher's note Springer Nature remains neutral with regard to jurisdictional claims in published maps and institutional affiliations. 